\title{
Modelo matemático para la dinámica de transmisión del VIH/SIDA en una población sexualmente activa
}

\section{A mathematical model representing HIV/AIDS transmission dynamics in a sexually-active population}

\author{
Mónica J. Mesa-Mazoํ, Juan G. Vergaño-Salazar ${ }^{1}$, Claudia E. Sánchez-Botero ${ }^{1}$ \\ y Aníbal Muñoz-Loaiza ${ }^{2}$
}

1 Facultad de Ciencias Básicas y Tecnologías, Universidad del Quindío. Armenia, Colombia. mjmm220@gmail.com,jgvs190@gmail.com, helecla@hotmail.com

2 Facultad de Educación, Universidad del Quindío. Armenia, Colombia. anibalml@ @otmail.com

Recibido 13 Mayo 2009/Enviado para Modificación 19 Diciembre 2009/Aceptado 31 Marzo 2010

\section{RESUMEN}

Se presenta un nuevo modelo que explica la dinámica de transmisión de la enfermedad del Síndrome de Inmunodeficiencia Adquirida entre parejas heterosexualmente activas. Se incluyen los supuestos, las variables, las ecuaciones diferenciales ordinarias, el análisis de sensibilidad del modelo y las estrategias de control. Los datos fueron obtenidos del Departamento Nacional de Estadísticas y se llevaron a cabo diferentes simulaciones sin control y con control en el sistema, utilizando el código del programa MAPLE. Los resultados obtenidos en las simulaciones permiten concluir que el control con preservativos es relevante en el modelo, así se lleve a cabo sobre la población de hombres o mujeres y sin importar la proporción de hombres y mujeres sexualmente activa.

Palabras Clave: SIDA, VIH, retrovirus, epidemia, infección, sensibilidad (fuente: DeCS,BIREME).

\section{ABSTRACT}

This article presents a new model explaining acquired immunodeficiency syndrome (AIDS) transmission dynamics amongst heterosexually active couples. It covers the assumptions made, the variables analysed, the model's sensitivity and the ordinary differential equations and control strategies used. The information was obtained from the Colombian state Statistics Department (DANE) and applied to different simulations in the system (with and without control), using the MAPLE programme code. Simulation results led to concluding that control using condoms was relevant in the model. It was not important if control were applied in women or men, nor was the percentage of sexually-active men and women.

Key Words: HIV, AIDS, retrovirus, epidemic, infection, sensitivity (source: $M e S H$, NLM). 
$\mathrm{E}$ 1 Síndrome de Inmunodeficiencia Adquirida (SIDA) es la fase final y más seria de la enfermedad producida por el Virus de Inmunodeficiencia Humana (VIH), el cual ha experimentado un rápido crecimiento en el mundo desde que fue reconocido por primera vez en 1981; su principal forma de contagio es por vía sexual (1), aunque otros medios de transmisión ocurren al compartir agujas hipodérmicas contaminadas con el virus (2), o durante la gestación, el parto y/o lactancia de mujeres infectadas (3) y en un bajo índice por transfusión sanguínea o por accidentes durante el tratamiento a pacientes que padecen la enfermedad.

El VIH produce una destrucción progresiva del sistema inmunológico, es decir, que frente a una enfermedad, el organismo del individuo afectado no puede defenderse de los microorganismos patógenos, lo que significa que una vez el virus penetra la célula, la persona crea anticuerpos que no le permiten responder a cualquier enfermedad debido a que estos son el medio de multiplicación del virus lo que conduce finalmente a la muerte del infectado. Se estima que más de 25 millones de personas en el mundo han muerto por esta enfermedad y que en la actualidad el número de infectados a nivel mundial es de 40 millones, lo que indica que se presenta una pandemia que ha concentrado la atención de numerosos científicos, médicos y organizaciones mundiales de la salud para contrarrestar el avance de este temible retrovirus (ONUSIDA).

Entender la dinámica del VIH/SIDA es complejo debido a la gran cantidad de variables y parámetros (4) asociados con la enfermedad tales como: La variación de las tasas de infección que dependen de cada uno de los grupos de alto riesgo (homosexuales, heterosexuales, drogadictos, etc.); el periodo de incubación es largo antes de que se manifiesten los síntomas y la infección depende de la evolución de la enfermedad en cada individuo (5).

Las más altas prevalencias de la enfermedad se encuentran en países en vía de desarrollo, donde el acceso a los programas de educación y salud es insuficiente, convirtiéndose en un contexto propicio para un rápido crecimiento de la epidemia en las próximas décadas.

Según ONUSIDA, desde su aparición en el mundo, a principios de los ochenta, la epidemia de VIH/SIDA ha sido la causa de más de 25 millones de muertes, en el último quinquenio se ha reportado un creciente número de casos a nivel global, con excepción del Caribe, donde en los últimos dos años se ha observado una relativa estabilización en las prevalencias de infección en población general. 
En América Latina, alrededor de 1,8 millones de personas (intervalo: 1,42,4 millones) están viviendo con el VIH/SIDA. En 2003, unas 59000 personas (intervalo: 65 000-110 000) fallecieron a causa del SIDA y otras 200000 (intervalo: 140000-340 000) contrajeron la infección (ONUSIDA).

En América Latina y el Caribe la propagación de la epidemia presenta dinámicas diferentes desde sus inicios. En los países del Caribe se ha propagado por contacto heterosexual, mientras que en la mayoría de países de América Latina, la epidemia afecta primordialmente a grupos específicos de poblaciones: homosexuales, consumidores de droga suministrada por vía intravenosa y a poblaciones vinculadas al comercio sexual, entre otros. En Colombia se observa una progresiva tendencia a la generalización de la epidemia, particularmente en las regiones donde la transmisión por contacto heterosexual predominaba ya desde comienzos de los noventa.

Los antecedentes mencionados con anterioridad infieren que se está frente a un problema social de gran envergadura, y cualquier esfuerzo por tratar de entender la dinámica de propagación de la enfermedad unido al análisis de trabajos epidemiológicos que permiten tener cierto control de la misma, sin duda redundará en planes estratégicos para la concientización y prevención del contagio del virus, es por este motivo que a continuación se presenta un modelo matemático en ecuaciones diferenciales ordinarias correspondiente a magnitudes promedio debido a que las variables estocásticas corresponde a un sistema no lineal acoplado, que permiten analizar el comportamiento de la enfermedad en una población heterosexualmente activa para visualizar mediante simulaciones los efectos y la sensibilidad de los parámetros que en él intervienen.

\section{Modelo matemático}

Modelo sin Control. Se considera un modelo tipo SI que describe la dinámica de transmisión del VIH-SIDA, síndrome de inmunodeficiencia adquirida, es decir, la población se divide en susceptibles (individuos que pueden ser infectados) e infectados (individuos portadores del VIH-SIDA). Se supone que los individuos infectados no se recuperan y mueren debido a la enfermedad y que la enfermedad se transmite solo vía contacto sexual en parejas heterosexuales, por este hecho, la población susceptible está conformada por la población sexualmente activa; la población total $N$ viene dada por $N=S+I$ donde la cantidad de individuos susceptibles está conformada por hombres y mujeres susceptibles denotados $X_{h}, X_{m}$, respectivamente, de igual forma la 
población de individuos infectados se conforma por $Y_{h}$, para hombres y $Y_{m}$ para mujeres. Se denota por $\sigma_{h}$ la proporción de hombres que entran a la población sexualmente activa, $\sigma_{m}$ proporción de mujeres que entran a la población sexualmente activa, $\mu$ tasa de mortalidad en personas susceptibles $\alpha, \varepsilon$ tasas de mortalidad por VIH/SIDA, para hombres y mujeres, respectivamente. No se considera mortalidad natural para las personas infectadas. Nótese que $\sigma_{h} N$ representa la fracción de hombres de la población que pasan a ser sexualmente activos, $\sigma_{m} N$ representa la fracción de mujeres de la población que pasan a ser sexualmente activas, $-\mu X_{h},-\mu X_{m}$, representan la fracción de individuos, hombres y mujeres respectivamente, que mueren de manera natural, - $\alpha Y_{h}$ representa la fracción de hombres que mueren por la enfermedad y $-\varepsilon Y_{m}$ representa la fracción de mujeres que mueren por la enfermedad.

Se denota con $\beta_{m}$ la tasa de infección de mujeres por contagio con hombres infectados, además $\frac{Y_{m}}{M}$ representa la fracción de mujeres que infectan a los hombres por contacto sexual, luego $-\beta_{m} \frac{Y_{m}}{M} X_{h}$ representa la fracción de hombres que pasan de ser susceptibles y llegan al estado infeccioso. Con $\beta_{h}$ se denota la tasa de infección de hombres por contagio con mujeres infectadas, además $\frac{Y_{h}}{H}$ representa la fracción de hombres que infectan a las mujeres por contacto sexual, luego $-\beta_{h} \frac{Y_{h}}{H} X_{m}$ representa la fracción de mujeres que pasan de ser susceptibles y llegan al estado infeccioso, como $\frac{d X_{h}}{d t} \mathrm{y} \frac{d X_{m}}{d t}$ es el cambio de la población de hombres y mujeres susceptibles, respectivamente, con respecto al tiempo, además $\frac{d Y_{h}}{d t}, \frac{d Y_{m}}{d t}$ es el cambio de la población de hombres y mujeres infectados, respectivamente, con respecto al tiempo.

Con estos supuestos y las anteriores notaciones, el modelo SI propuesto para la dinámica del VIH-SIDA es

$$
\begin{gathered}
\frac{d X_{h}}{d t}=\sigma_{h} N-\beta_{m} \frac{Y_{m}}{M} X_{h}-\mu X_{h} \\
\frac{d Y_{h}}{d t}=\beta_{m} \frac{Y_{m}}{M} X_{h}-\alpha Y_{h} \\
\frac{d X_{m}}{d t}=\sigma_{m} N-\beta_{h} \frac{Y_{h}}{H} X_{m}-\mu X_{m} \\
\frac{d Y_{m}}{d t}=\beta_{h} \frac{Y_{h}}{H} X_{m}-\varepsilon Y_{m}
\end{gathered}
$$

Para mostrar la dinámica de transmisión de la enfermedad se realizan algunas simulaciones en un intervalo de tiempo $t$ en años, donde $t \in[0 ; 20]$; con los siguientes parámetros, $\beta_{m}=0,6, \beta_{h}=0,4, \alpha=\varepsilon=0,054, \mu=0,0138$; el modelo es sensible a los valores de $\sigma_{h}, \sigma_{m}$, donde $0<\sigma_{h}<1$ y $0<\sigma_{m}<1$. 
Si la proporción de hombres y mujeres que entran a ser parte de la población sexualmente activa son iguales, es decir $\sigma_{h},=\sigma_{m}=0,025$ se observa que la población de hombres infectados crece en los primeros quince años; la dinámica de la enfermedad se muestra en la Figura 1. Si la proporción de hombres que entran a ser parte de la población sexualmente activa es $\sigma_{h}=0,025$ y la proporción de mujeres que entran a ser parte de la población sexualmente activa es $\sigma_{m}=0,85$, se muestra que la población de mujeres infectadas crece sin estabilizarse en esos veinte años; la dinámica de la enfermedad se muestra en la Figura 2. Si se toma a $\sigma_{h}=0,85$ y a $\sigma_{m}=0,025$; se tiene que la población de hombres infectados crece en ese mismo intervalo de tiempo; la dinámica se muestra en la Figura 3. Si $\sigma_{h}=\sigma_{m}=0,85$, se observa que tanto la población de hombres infectados como de mujeres infectadas crece en los veinte años; la dinámica se obtiene en la Figura 4.

Para las gráficas,

$X_{h}:=$ Continua, $Y_{h}:=$ Rayada, $X_{m}:=$ Puntead $, Y_{m}:=$ Raya - punto

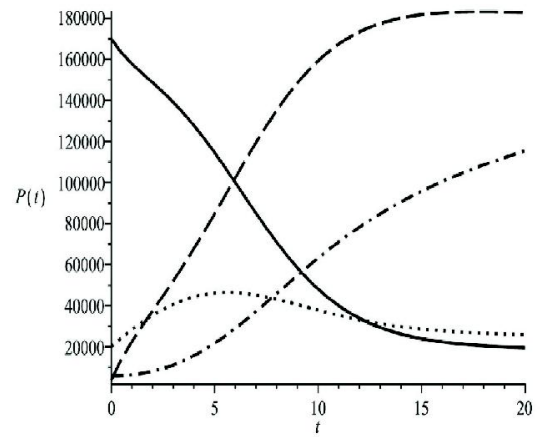

Figura. 1

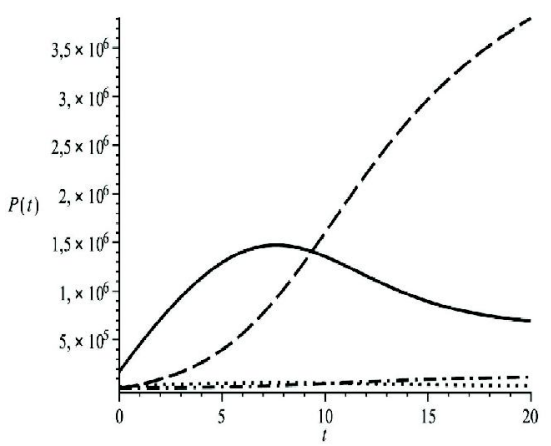

Figura. 3

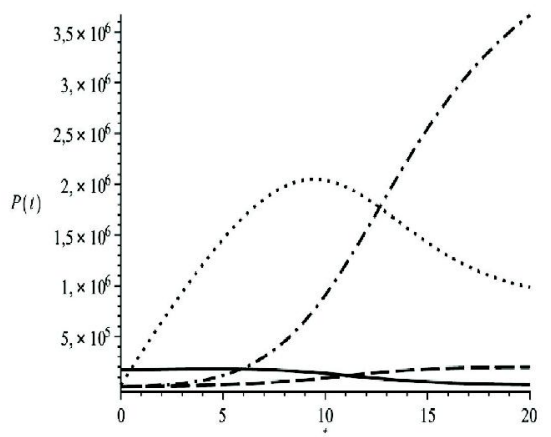

Figura. 2

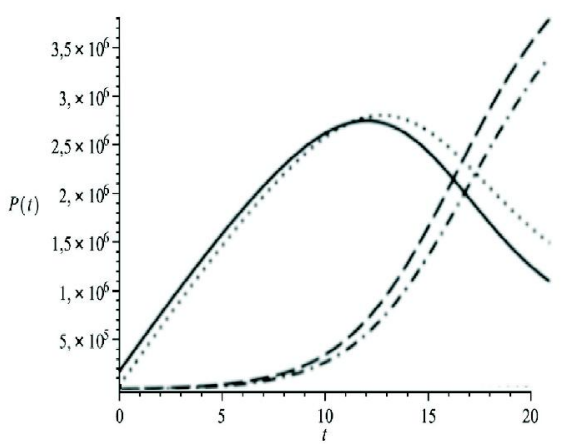

Figura. 4 
Extensión del Modelo, Estrategia de Control. Se considera que una proporción de individuos es protegida, es decir, se tiene en cuenta el uso de preservativos tanto en hombres como en mujeres; sean $f$ la fracción de hombres sexualmente activos que se protegen con el uso de preservativos y $g$ la fracción de mujeres sexualmente activas que utilizan algún medio de protección; sea (1- $f$ ) la fracción de hombres que no se protegen, luego $-\beta_{m} \frac{Y_{m}}{M}(1-f) X_{h}$ es la fracción de hombres que no se protegen y son infectados por mujeres, además, (1-g) representa la fracción de mujeres que no se protegen, por lo tanto $-\beta_{h} \frac{Y_{h}}{H}(1-g) X_{m}$ es la fracción de mujeres que no se protegen y son infectadas por hombres; los demás supuestos del modelo son los mismos. Con los supuestos y notaciones anteriores, el modelo con estrategia de control esta dado por el siguiente sistema de ecuaciones diferenciales ordinarias:

$$
\begin{aligned}
\frac{d X_{h}}{d t} & =\sigma_{h} N-\beta_{m} \frac{Y_{m}}{M}(1-f) X_{h}-\mu X_{h} \\
\frac{d Y_{h}}{d t} & =\beta_{m} \frac{Y_{m}}{M}(1-f) X_{h}-\alpha Y_{h} \\
\frac{d X_{m}}{d t} & =\sigma_{m} N-\beta_{h} \frac{Y_{h}}{H}(1-g) X_{m}-\mu X_{m} \\
\frac{d Y_{m}}{d t} & =\beta_{h} \frac{Y_{h}}{H}(1-g) X_{m}-\varepsilon Y_{m}
\end{aligned}
$$

Para mostrar la dinámica de transmisión de la enfermedad en un intervalo de veinte años, cuando una fracción de la población se protege con uso del preservativo, se toman en cuenta los siguientes parámetros, $\beta_{m}=0,6, \beta_{h}=0,4$, $\alpha=\varepsilon=0,054, \mu=0,0138$; el modelo es sensible a los valores de $\sigma_{h}, \sigma_{m}, f, g$, donde $0<\sigma_{h}<1$ y $0<\sigma_{m}<1$ y $0<f<1 ; 0<g<1$. Si la proporción de hombres y mujeres que entran a ser parte de la población sexualmente activa son iguales, es decir $\sigma_{h}=\sigma_{m}=0,025$ y los controles asumen valores $f=g$ $=0,1$ se observa que la población de hombres infectados crece en los primeros quince años; la dinámica de la enfermedad se observa en la Figura 5; si la proporción de hombres que entran a ser parte de la población sexualmente activa es $\sigma_{h}=0,025$ y la proporción de mujeres que entran a ser parte de la población sexualmente activa, $\sigma_{m}=0,85$ y los controles asumen los valores $f=0,1$ y $g=0,8$; debido a que el control que se ejerce con mayor fuerza sobre una de las poblaciones, se tiene que la población de mujeres susceptibles en los veinte años aumenta; la dinámica de la enfermedad se muestra en la Figura 6; si se hace $\sigma_{h}=0,025, \sigma_{m}=0,85$ y los controles asumen valores $f=0,8$ y $g=0,1$; se observa que después de los primeros quince años, la población de mujeres infectadas empieza a crecer; la dinámica de la enfermedad se observa en la Figura 7; si la proporción de hombres que entran a ser parte de la población sexualmente activa es $\sigma_{h}=0,85$ y la proporción de mujeres que entran a ser parte de la población 
sexualmente activa es $\sigma_{m}=0,025$, y además los controles tienen valores $f=0,1$ y $g=0,8$, se tiene que la población de hombres infectados aumenta debido al bajo control que se ejerce sobre ellos; la dinámica de la enfermedad se muestra en la Figura 8; para $\sigma_{\mathrm{h}}=0,85, \sigma_{m}=0,025$ y para los controles $f=0,8$ y $g=0,1$, se observa que después de los primeros diez años, la población de hombres infectados empieza a crecer; el comportamiento de la enfermedad se puede ver en la Figura 9; por último, si los valores son $\sigma_{h}=0,85, \sigma_{m}$ y $f=0,8=g$ debido al alto control en ambas poblaciones se tiene que tanto hombres como mujeres susceptibles aumente; la dinámica de la población se muestra en la Figura 10. Para las gráficas,

Entender la dinámica de una enfermedad permite hacer un análisis para determinar los factores más sensibles de la misma, lo que conduce a tener argumentos para la elaboración de estrategias de control sobre ellos. El VIH/SIDA es un virus que no deja de sorprender a la comunidad científica en general, debido a la cantidad de factores que contribuyen al aumento de seropositivos, pese a que el modelo presentado suprime muchos de ellos; no obstante, la dinámica modelada advierte que los parámetros más sensibles están en las tasas $\sigma_{m \text { y }} \sigma_{m}$ Se advierte que los datos utilizados para hacer las simulaciones corresponden a datos obtenidos del Departamento Nacional de Estadísticas; sin embargo, en Colombia hace falta tener un registro más detallado de las personas que padecen la enfermedad y el cual se ha depurado constantemente, esto sería un gran insumo para un modelado más real del fenómeno; Las simulaciones se llevan a cabo en un intervalo de tiempo de 20 años debido a que la enfermedad normalmente se desarrolla en este periodo; después de realizadas las simulaciones en este tiempo, se observa de la Figura 1 que la población de hombres susceptibles disminuye rápidamente y la población de hombres infectados aumenta de igual forma, mientras que en las mujeres se presenta un pequeño cambio tanto en susceptibles como en infectadas; de la Figura 2, el crecimiento de las mujeres infectadas es mayor que el de los hombres infectados el cual presenta un cambio mínimo. De esta forma solo puede verse una dinámica en las mujeres, además en la Figura 3 ocurre todo lo contrario dado que la dinámica solo ocurre en los hombres; para la Figura 4, ambas poblaciones, tanto susceptibles como infectados en hombres y mujeres, presentan la misma dinámica; se encuentra que en la Figura 5, la dinámica es igual a la obtenida en la Figura 1. Debido a que el control que se ejerce en ambas poblaciones es muy bajo; por último, en las Figuras $6,7,9$, se observa que el control es efectivo sin importar si se hace sobre la población de hombres o de mujeres y sin ser susceptible a la tasa de ingreso de las poblaciones 
$X_{h}:=$ Continua $, Y_{h}:=$ Rayada, $X_{m}:=$ Punteada, $Y_{m}:=$ Raya - punto

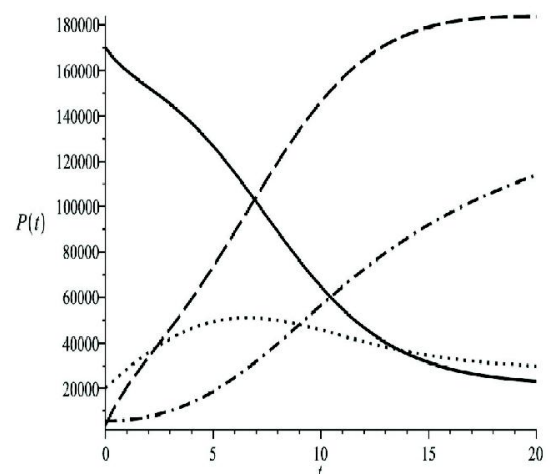

Figura. 5

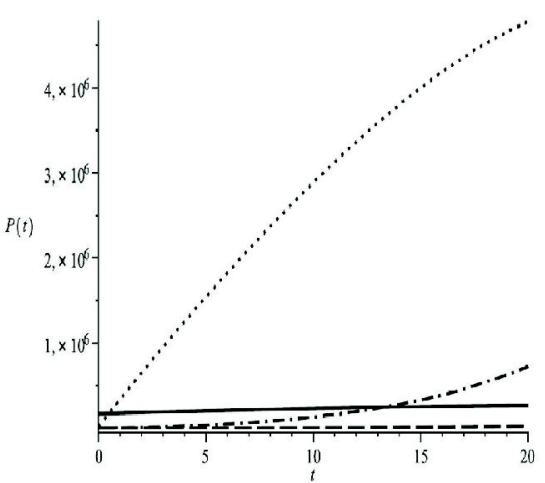

Figura. 7

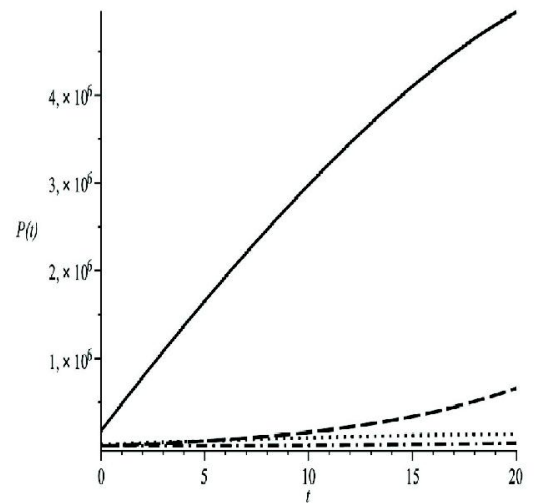

Figura. 9

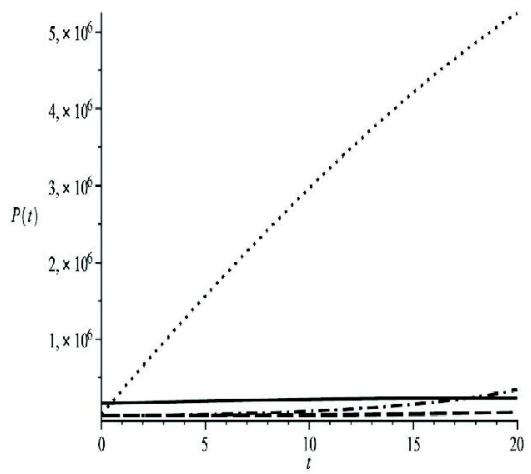

Figura. 6

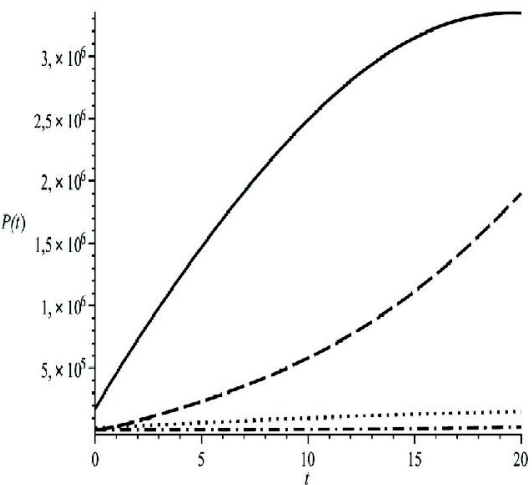

Figura. 8

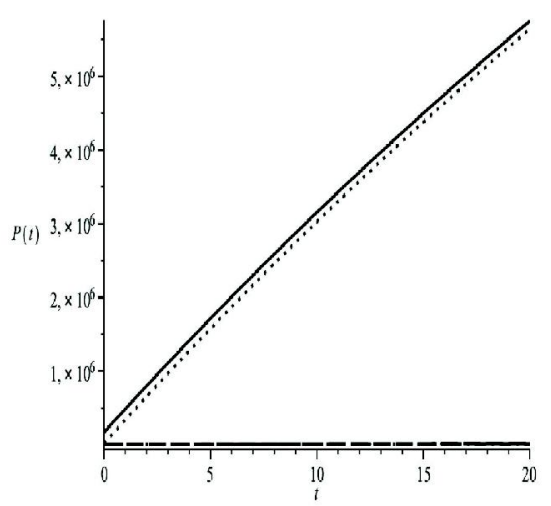

Figura. 10 


\section{REFERENCIAS}

1. Hsieh Y, Wang Y. Basic Reproduction Number for HIV Model Incorporating Commercial Sex and Behavoir Change. Bulletin of Mathematical Biology. 2006; 68; 551-575.

2. Greenhalgh $D$, Lewis $F$. The general mixing of addicts and needles in a variable-infectivity needle-sharing environment. Bulletin of Mathematical Biology. 2002; 44; 561-598.

3. Culshaw RV, Ruan S, Webb G. A mathematical model of cell-to-cell spread of HIV-1 that includes a time delay. Bulletin of Mathematical Biology. 2003; 46, 425-444.

4. Hulin W, Haihong Z, Hongyu M, Perelson AS. Parameter identifiability and Estimation of HIV/ AIDS Dynamic Model; Bulletin of Mathematical Biology. 2008; 70; 785-799.

5. Culshaw RV, Ruan S, Spiteri RJ. Optimal HIV treatment by maximising immune response. Bulletin of Mathematical Biology. 2004; 48, 545-562.

6. Knolle H. A discrete branching process model for the spread of HIV via steade sexual partnerships. Bulletin of Mathematical Biology. 2004; 48, 423-443.

7. Adams BM, Banks HT, Davidian M, Rosenberg ES. Estimation and Prediction With HIVTreatment Interruption Data. Bulletin of Mathematical Biology. 2007; 69; 563-584.

8. González PA, Quintero JR. Un modelo de VHI-SIDA con reinfección. Matemáticas. Enseñanza Universitaria, Revista de la ERM. 2002; 10; 63-79.

9. Amador R. Safety and inmunologenicity of the synthetic malaria vaccine Spf66 in a large .eld trial. The Journal of Infectious Diseases. 1992; 166: 139-144.

10. Keener J, Sneyd J. Mathematical Physiology. Editors, J.E. Marsden; 1991.

11. Edelstein-Keshet L. Mathematical Models in Biology. Mc Graw-Hill; 1988. 12. Perko, Lawrence. Differential Equations end Dinamical Sistems. Second Edition, Springer; 1996. 